\title{
Synchronization Between Two Different Switched Chaotic Systems By Switching Control
}

\author{
Li Ming DU ${ }^{1, a}$, Feng Ying WANG ${ }^{1}$, Jie DONG ${ }^{1}$ and Zheng Yu LI ${ }^{1}$ \\ ${ }^{1}$ Faculty of Information \& Control Engineering, Shenyang Jianzhu University, Shenyang 110168
}

\begin{abstract}
This paper is concerned with the synchronization problem of two different switched chaotic systems, considering the general case that the master-slave switched chaotic systems have uncertainties. Two basic problems are considered: one is projective synchronization of switched chaotic systems under arbitrary switching; the other is projective synchronization of switched chaotic systems by design of switching when synchronization cannot achieved by using any subsystems alone. For the two problems, common Lyapunov function method and multiple Lyapunov function method are used respectively, an adaptive control scheme has been presented, some sufficient synchronization conditions are attainted, and the switching signal is designed . Finally, the numerical simulation is provide to show the effectiveness of our method.
\end{abstract}

\section{Introduction}

Chaos is very interesting nonlinear phenomenon and has been intensively studied in the last three decades [1-3]. In particular, chaos synchronization has become one of the hot issues since Pecora and Carroll introduced a method to synchronize two identical chaotic systems with different initial conditions[4]. For it's potential applications in secure communication, chemical and biological systems, information science and many other fields, lots of researches on the synchronization of coupled chaotic dynamical systems have been done and many theoretical and experimental results have been obtained[5-19]. Such as feedback control methods[5-7], backstepping design technique[8], impulsive control method[9], fuzzy sliding mode control method [10], adaptive method[11,12] and other kind of synchronization[13-19].

Most studies are assumed that the master and the slave systems are single accurate mathematical model of the chaotic system with same dynamical structures. However, it is difficult to find two exactly identical chaotic systems. On the other hand, the master and slave chaotic systems may change very quickly-even by jumping or switching. To the best of the author's knowledge, no synchronization criteria have been reported for this kind of studies. Obviously, synchronization between two different switched chaotic systems are more interesting and worth researching. For example, it can improves safety features of chaos synchronization secret communication while applying in secret communication because of the richness of selection in switched chaotic systems which achieves the variety of transmisson signal.

\footnotetext{
${ }^{\mathrm{a}}$ Corresponding author: duliboy@163.com
}

This paper studies the projective synchronization problems between different switched chaotic systems with uncertainty. By utilizing switching control theory and adaptive control technology, an adaptive control scheme has been presented, some sufficient synchronization conditions are attainted, and the switching signal is designed based on multiple Lyapunov function method.

\section{Problem Formulation}

Consider the master-slave switched chaotic systems is described as follow

$$
\begin{aligned}
& \dot{x}=A_{\sigma} x+B_{\sigma} f_{\sigma}(t, x, \theta), \\
& \dot{y}=A_{\sigma} y+B_{\sigma}\left(g_{i}(t, y, \tilde{\theta})+u_{\sigma}(t)\right),
\end{aligned}
$$

where $t \in R^{+}, x \in R^{n}$ is the state variable of master system, $y \in R^{n}$ is the state variable of the slave system, $\sigma: R^{+} \rightarrow M=\{1,2, \ldots, m\}$ is the switching signal, $A_{i}, B_{i}, i \in M$ denote known matrices with appropriate dimensions of the $i$ th subsystem, $\theta, \tilde{\theta}$ is constant matrix which may be unknown, $f_{i}(t, x, \theta): J \times R^{n} \times R^{n \times n} \rightarrow R^{n}$ with $f_{i}(t, 0, \theta) \equiv 0 \quad$ and $g_{i}(t, x, \tilde{\theta}): J \times R^{n} \times R^{n \times n} \rightarrow R^{n} \quad$ with $\quad g_{i}(t, 0, \tilde{\theta}) \equiv 0 \quad$ are continuous vector-value function, $u_{i}(t), \quad i \in M$ is the controller of the ith subsystem. It is easy to see that many chaotic systems can be described as the subsystem form 
of (1) and (2), such as the Lorenz system, Chen's system, Liu system, and Chua's circuit.

Definition 1. For the given master system $\dot{x}_{i}=f_{i}\left(x_{i}\right)$, and slave system $\dot{y}_{i}=g_{i}\left(y_{i}\right)$, where $i=1,2, \ldots, n, \quad x_{i} \in R, \quad y_{i} \in R$, We called the master and slave systems achieve projective synchronization if there exist scaling factors $h$, which satisfied $\lim \left\|x_{i}-h y_{i}\right\|=0$.

The error dynamical system between Eqs. (1) and (2) can be expressed as

$$
\dot{e}=A_{\sigma} e+B_{\sigma}\left(g_{\sigma}(t, y, \tilde{\theta})-h f_{\sigma}(t, x, \theta)+u_{\sigma}(t)\right),
$$

where $e=y-h x$ is the synchronization error. So the synchronization problem is now replaced by the problem of stabilizing the system (3).

Assumption 1: The nonlinear part of the system (1) and (2) $f: J \times R^{n} \times R^{n \times n} \rightarrow R^{n}$ satisfies the following condition

$$
\left\|g_{i}(t, y, \tilde{\theta})-h f_{i}(t, x, \theta)\right\| \leq l\|y-h x\|, \quad i \in M,
$$

It is easy to see that the synchronization problem turn into the stabilization problem of the system (3).

\section{Common Lyapunov function method}

In this section, we will discuss the projective synchronization between different switched chaotic systems by common Lyapunov function method. First, we design the controllers as the form of (5).

$$
u_{i}=K_{i} e-\frac{1}{2} \lambda r B_{i}^{T} P e, \lambda \in R_{+}, K_{i} \in R^{n \times n}, i \in M,
$$

Where $r$ represents the dynamical estimate of $l^{2}$ in assumption (4), and The estimate $r(t)$ is updated according to the following algorithm:

$$
\dot{r}=\frac{1}{2} \lambda\|e\|^{2}, \lambda \in R^{+},\|e\|=\left(\sum_{i=1}^{n} e^{2}\right)^{\frac{1}{2}} .
$$

Under control law (5) and the adaptation algorithm (6), the ith subsystem of (3) can be characterized as follow form:

$$
\begin{aligned}
& \dot{e}=\left(A_{i}+B_{i} K_{i}-\frac{1}{2} \lambda r B_{i} B_{i}^{T} P\right) e+B_{i}\left(g_{i}(t, y, \tilde{\theta})-h f_{i}(t, x, \theta)\right), \\
& \dot{r}=\frac{1}{2} \lambda\|e\|^{2},
\end{aligned}
$$

where $e=y-h x$, then we discuss the asymptotic stabilization of the system (7).

Theorem 1. consider the error system (7) of master system (1) and the controlled slave system (2) satisfying Assumption 1. If there exist a positive definite matrix $P$, a matrix $K_{i}$ and a positive constant $\lambda$ such that the following LMI

$$
\left(A_{i}+B_{i} K_{i}\right)^{T} P+P\left(A_{i}+B_{i} K_{i}\right)+\lambda^{-1} I<0, i \in M,
$$

is satisfied. then the error system (7) is global asymptotic stable with the adaptive controller(5) under arbitrary switching, that is to say, the master-slave systems achieve projective synchronization.

Proof. Construct a common Lyapunov function in the form of

$$
V=e^{T} P e+\frac{1}{\lambda}\left(l^{2}-r\right)^{2},
$$

The derivative of Lyapunov function along trajectory of each subsystem in system (7) is

$$
\begin{aligned}
\dot{V}= & \dot{e}^{T} P e+e^{T} P \dot{e}-\frac{2}{\lambda}\left(l^{2}-r\right) \dot{r} \\
= & e^{T}\left(P\left(A_{i}+B_{i} K_{i}\right)+\left(A_{i}+B_{i} K_{i}\right)^{T} P\right) e-\lambda \hat{r} e^{T} P B_{i} B_{i}^{T} P e \\
& +2 e^{T} P B_{i}\left(g_{i}(t, y, \tilde{\theta})-h f_{i}(t, x, \theta)\right)-\frac{2}{\lambda}\left(l^{2}-r\right) \dot{r} \\
\leq & e^{T}\left(P\left(A_{i}+B_{i} K_{i}\right)+\left(A_{i}+B_{i} K_{i}\right)^{T} P\right) e-\lambda r\left\|B_{i}^{T} P e\right\|^{2} \\
& +2\left\|B_{i}^{T} P e\right\|\left\|g_{i}(y)-h f_{i}(x)\right\|-\frac{2}{\lambda}\left(l^{2}-r\right) \dot{r} \\
\leq & e^{T}\left(P\left(A_{i}+B_{i} K_{i}\right)+\left(A_{i}+B_{i} K_{i}\right)^{T} P\right) e-\lambda r\left\|B_{i}^{T} P e\right\|^{2} \\
& +2 l\left\|B_{i}^{T} P e\right\|\|e\|-\frac{2}{\lambda}\left(l^{2}-r\right) \dot{r} \\
\leq & e^{T}\left(P\left(A_{i}+B_{i} K_{i}\right)+\left(A_{i}+B_{i} K_{i}\right)^{T} P\right) e-\lambda r\left\|B_{i}^{T} P e\right\|^{2} \\
& +\lambda l^{2}\left\|B_{i}^{T} P e\right\|^{2}+\frac{1}{\lambda}\|e\|^{2}-\frac{2}{\lambda}\left(l^{2}-r\right) \dot{r} .
\end{aligned}
$$

By substituting Eq. (6) into Eq. (10), we have

$$
\dot{\mathrm{V}} \leq \mathrm{e}^{\mathrm{T}}\left(\left(A_{i}+B_{i} K_{i}\right) P+\left(A_{i}+B_{i} K_{i}\right)^{T} P+\lambda^{-1} I\right) e<0 .
$$

According to the stability theory of switching system, error system (7) is asymptotically stable with arbitrary switching conditions, i.e. $\|e(t)\|=\|y(t)-h x(t)\| \rightarrow 0$ as $t \rightarrow \infty$. It is obviously that that the master-slave systems achieve projective synchronization, which complete the proof.

\section{Multiple Lyapunov function method}

In this section, we will study the case that none of subsystems can achieve synchronization. For this kind of condition, the common Lyapunov function could not get, so we discuss how to realize synchronization by the suitable design of switching ruler between sub chaotic systems and give a multiple Lyapunov function methos.

Theorem 2. Under the assumption 1, If there exist $\delta_{i j} \geq 0 \quad\left(\delta_{i j} \leq 0\right)$ and positive defined matrix $P_{i}$ such that LIMs

$$
\begin{gathered}
\left(A_{i}+B_{i} K_{i}\right)^{T} P_{i}+P_{i}\left(A_{i}+B_{i} K_{i}\right)+\lambda^{-1} I+\sum_{j=1}^{m} \delta_{i j}\left(P_{j}-P_{i}\right)<0 \\
i=1,2, \ldots, m
\end{gathered}
$$


hold, then synchronization between master systems (1) and slave systems(2) is achieved under the switching controller (5) with switching signal

$$
\sigma(t)=i, \text { if } e(t) \in \bar{\Omega}_{i}, \quad i \in M
$$

where

$$
\Omega_{i}=\left\{e \in R^{n} \mid e^{T}\left(P_{j}-P_{i}\right) e \geq 0, \forall j \in M\right\}
$$

and $\overline{\mathrm{W}}=\mathrm{W}, \cdots, \bar{\Omega}_{i}=\Omega_{i}-\bigcup_{j=1}^{i-1} \bar{\Omega}_{j}, \cdots, \quad \overline{\mathrm{W}}_{m}=\mathrm{W}_{m}-\bigcup_{j=1}^{m-1} \overline{\mathrm{W}}_{j}$.

Proof. Let $\delta_{i j} \geq 0$,then for any $e \in R^{n} \backslash\{0\}$, exist a $i \in M$ to make $e^{T}\left(P_{j}-P_{i}\right) e \geq 0, " j \hat{\mathrm{I}} M$, according to (12), we get

$$
\left(A_{i}+B_{i} K_{i}\right)^{T} P_{i}+P_{i}\left(A_{i}+B_{i} K_{i}\right)+\lambda^{-1} I<0, i \in M,
$$

Then, for any $i \in M$, let construct Lyapunov function as the form of

$$
V(t)=e^{T} P_{i} e+\frac{1}{\lambda}\left(l^{2}-r\right)^{2},
$$

The derivative of $i$ th Lyapunov function along the trajectory of (7) is

$$
\begin{aligned}
\dot{V}(t) & =\dot{e}^{T} P_{i} e+e^{T} P_{i} \dot{e}-\frac{2}{\lambda}\left(l^{2}-r\right) \dot{r} \\
= & e^{T}\left(P_{i}\left(A_{i}+B_{i} K_{i}\right)+\left(A_{i}+B_{i} K_{i}\right)^{T} P_{i}\right) e-\lambda \hat{r} e^{T} P_{i} B_{i} B_{i}^{T} P_{i} e \\
& +2 e^{T} P_{i} B_{i}\left(g_{i}(t, y, \tilde{\theta})-h f_{i}(t, x, \theta)\right)-\frac{2}{\lambda}\left(l^{2}-r\right) \dot{r} \\
\leq & e^{T}\left(P_{i}\left(A_{i}+B_{i} K_{i}\right)+\left(A_{i}+B_{i} K_{i}\right)^{T} P_{i}\right) e-\lambda r\left\|B_{i}^{T} P_{i} e\right\|^{2} \\
& +2 l\left\|B_{i}^{T} P_{i} e\right\|\|e\|-\frac{2}{\lambda}\left(l^{2}-r\right) \dot{r} \\
\leq & e^{T}\left(P_{i}\left(A_{i}+B_{i} K_{i}\right)+\left(A_{i}+B_{i} K_{i}\right)^{T} P_{i}\right) e-\lambda r\left\|B_{i}^{T} P_{i} e\right\|^{2} \\
& +\lambda l^{2}\left\|B_{i}^{T} P_{i} e\right\|^{2}+\frac{1}{\lambda}\|e\|^{2}-\frac{2}{\lambda}\left(l^{2}-r\right) \dot{r} . \\
\leq & \mathrm{e}^{\mathrm{T}}\left(\left(A_{i}+B_{i} K_{i}\right) P_{i}+\left(A_{i}+B_{i} K_{i}\right)^{T} P_{i}+\lambda^{-1} I\right) e .
\end{aligned}
$$

From (15), it is easy to see that error system (7) is asymptotically stable under switching ruler(13), and the theorem 2 is proved.

\section{Numerical Simulation}

In this section we give numerical experiments on the problems of projective synchronization of master system(1) and slave system(2) to illustrate the validity of Theorems.

Let take Lorzen and Genesio system as the subsystems of master switched chaotic system and Liu and Rossler system as the subsystems of slave switched chaotic sysem. The Lorenz system is a very typical chaotic system, it is presented as:

$$
\left\{\begin{array}{l}
\dot{x}_{1}=a_{1}\left(x_{2}-x_{1}\right) \\
\dot{x}_{2}=a_{2} x_{1}-x_{2}-x_{1} x_{3} \\
\dot{x}_{3}=-a_{3} x_{3}+x_{1} x_{2}
\end{array}\right.
$$

where $x$ is the state variable of master system, $a_{1}, a_{2}, a_{3}$ are the parameters of system, If we choose $a_{1}=10, a_{2}=28, a_{3}=8 / 3$, the system stays in chaotic state. As we assume that the initial condition, $x_{1}(0)=0.1$ , $x_{2}(0)=0.5, x_{3}(0)=0.3$, chaotic attractor is shown in Figure 1.

Genesio system is described as:

$$
\begin{aligned}
& \dot{x}_{1}=x_{2} \\
& \dot{x}_{2}=x_{3} \\
& \dot{x}_{3}=-\hat{a}_{1} x_{1}-\hat{a}_{2} x_{2}-\hat{a}_{3} x_{3}+x_{1}^{2}
\end{aligned},
$$

where $\hat{a}_{1}=6, \hat{a}_{2}=2.92, \hat{a}_{3}=1.2$, and the initial point of system $x_{1}(0)=0.1, x_{2}(0)=0.5, x_{3}(0)=0.3$, the chaotic attractor of Genesio system is displayed as Figure 2.

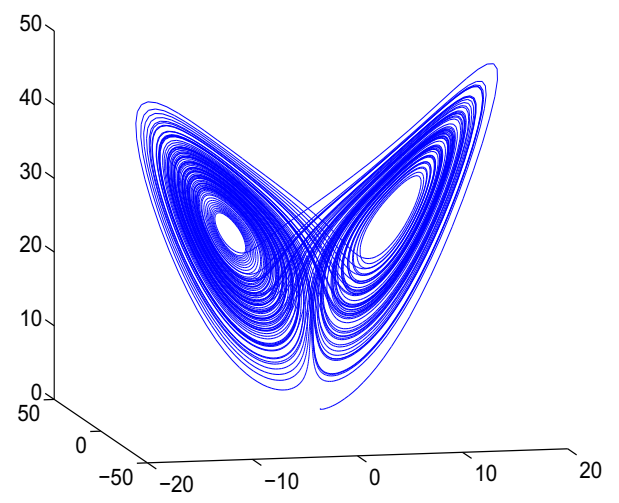

Figure 1. A chaotic attractor of Lorenz system

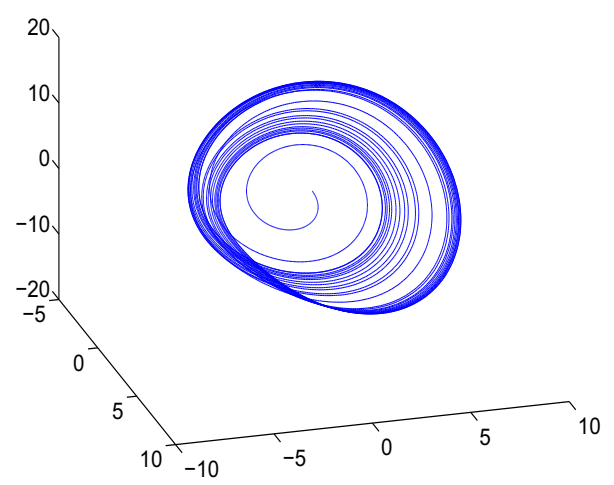

Figure 2. A chaotic attractor of Genesio system

Liu system is another typical chaotic system model, which is described as:

$$
\left\{\begin{array}{l}
\dot{\mathrm{y}}_{1}=\mathrm{b}_{1}\left(\mathrm{y}_{2}-\mathrm{y}_{1}\right) \\
\dot{\mathrm{y}}_{2}=\mathrm{b}_{2} \mathrm{y}_{1}-\mathrm{b}_{4} \mathrm{y}_{1} \mathrm{y}_{3} \\
\dot{\mathrm{y}}_{3}=-\mathrm{b}_{3} \mathrm{y}_{3}+\mathrm{b}_{5} \mathrm{y}_{1}^{2}
\end{array},\right.
$$


where $y$ is the state variables of slave system, $b_{1}, b_{2}, b_{3}$, $\mathrm{b}_{4}, \mathrm{~b}_{5}$ denote the parameters of system, if $\mathrm{b}_{1}=10, \mathrm{~b}_{2}=28, \quad \mathrm{~b}_{4}=1, \mathrm{~b}_{5}=4 \mathrm{~b}_{3}=8 / 3$, the system stands in chaos, as the initial point of system $\mathrm{y}_{1}(0)=5, \mathrm{y}_{2}(0)=-6, \mathrm{y}_{3}(0)=4$, its chaotic attractor is displayed as Figure 3.

Rossler system has plentiful chaotic features and is described by:

$$
\left\{\begin{array}{l}
\dot{\mathrm{y}}_{1}=-\mathrm{y}_{2}-\mathrm{y}_{3} \\
\dot{\mathrm{y}}_{2}=\mathrm{y}_{1}+\hat{\mathrm{b}}_{2} \mathrm{y}_{2} \\
\dot{\mathrm{y}}_{3}=\hat{\mathrm{b}}_{1}+\mathrm{y}_{3}\left(\mathrm{y}_{1}-\hat{\mathrm{b}}_{3}\right)
\end{array}\right.
$$

where $\hat{b}_{1}, \hat{b}_{2}, \hat{b}_{3}$, denote the parameters of system, if $\hat{b}_{1}=0.2, \hat{b}_{2}=0.2, \hat{b}_{3}=5.7$, the system is chaotic, as the initial point of system $\mathrm{y}_{1}(0)=5, \mathrm{y}_{2}(0)=-6, \mathrm{y}_{3}(0)=4$, its chaotic attractor is displayed as Figure 4.

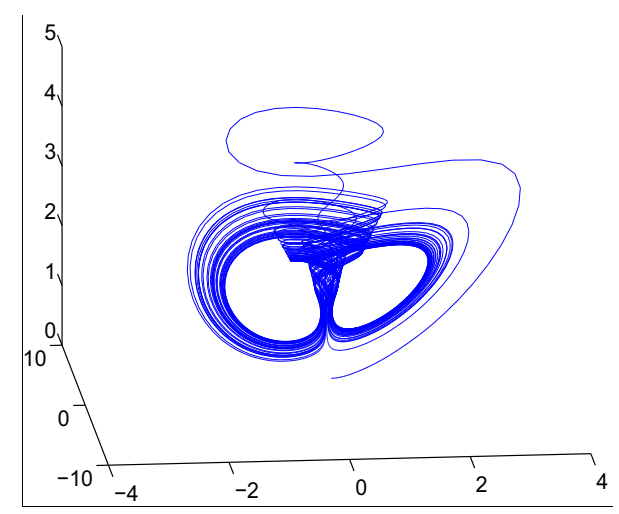

Figure 3. A chaotic attractor of Liu system

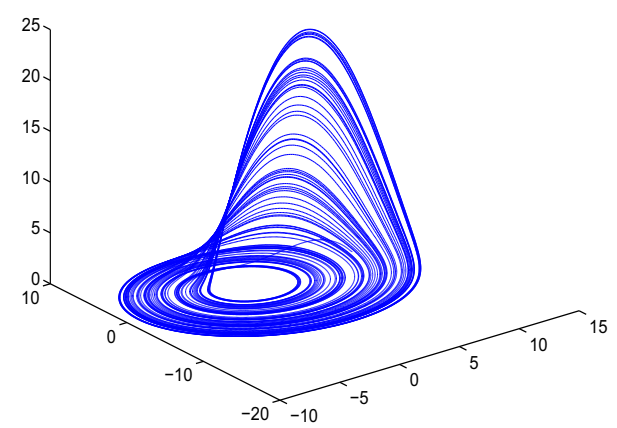

Figure 4. A chaotic attractor of Rossler system

It is easy to see that the system (17) (18)and system (19) (20)can be rewrited as the form of Eqs. (1) and (2), if we take

$$
\begin{array}{cc}
A_{1}=\left[\begin{array}{ccc}
-10 & 10 & 0 \\
28 & 0 & 0 \\
0 & 0 & -8 / 3
\end{array}\right], & A_{2}=\left[\begin{array}{ccc}
0 & 1 & 0 \\
0 & 0 & 1 \\
-6 & -2.92 & -1.2
\end{array}\right], \\
B_{1}=B_{2}=\left[\begin{array}{ccc}
1 & 0 & 0 \\
0 & 1 & 0 \\
0 & 0 & 1
\end{array}\right], & K_{1}=\left[\begin{array}{ccc}
-3 & 0 & 0 \\
0 & -2 & 0 \\
0 & 0 & -5
\end{array}\right], \\
K_{2}=\left[\begin{array}{ccc}
-4 & 0 & 0 \\
0 & -3 & 0 \\
0 & 0 & -2
\end{array}\right], & h=3, \lambda=2,
\end{array}
$$

then we can get the positive matrix

$$
P=\left[\begin{array}{llc}
0.8780 & -0.3505 & 0 \\
-0.0505 & 0.1812 & 0 \\
0 & 0 & 3.5787
\end{array}\right],
$$

which satisfied the inequality (8). If the initial state of system $x(0)=\left(\begin{array}{lll}0.1 & 0.3 & 0.5\end{array}\right), y(0)=\left(\begin{array}{lll}5 & -6 & 4\end{array}\right), r(0)=8$, and put in control as $\mathrm{t}>5$, the simulation result is displayed as Figure 5.

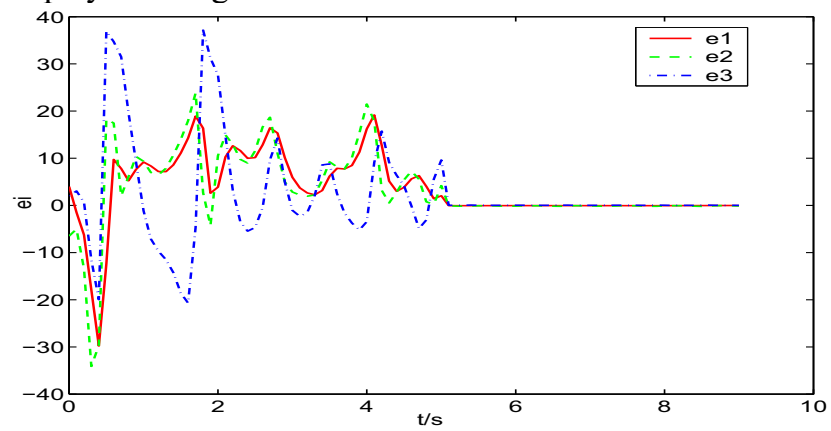

Figure 5. The evolution of the error vector

From Figure 5, we can clearly see that the error state of system reaches zero quickly with adaptive controller under arbitrary switching, which demonstrates the validity of our results.

Remark 1. In the paper, if we take $h=1$, then the two different switched chaotic systems will get complete synchronization with the adaptive controller under arbitrary switching, and if we take $h=-1$, the masterslave switched chaotic systems will get antisynchronization under arbitrary switching.

\section{CONCLUSIONS}

In the paper, we present a novel method to solve the problem of projective synchronization between different switched chaotic systems, By adopting common Lyapunov function method and multiple Lyapunov function method, an adaptive controller is achieved, which realize the projective synchronization between the master-slave system with different switched chaotic systems under arbitrary switching and designed switching. Numerical simulation indicates the accuracy of the results. 


\section{Acknowledgement}

This work was supported by the National Natural Science Foundation of China under Grants 61272253 and Shenyang Jianzhu University Subject Culvert Project XKHY2-72.

\section{References}

1. A.L. Fradkov, R. J. Evans, Annual Reviews in Control , 29(1), 33-56, (2005).

2. Y.Chang, G.Chen, Chaos, Solitons \& Fractals, 27(1), 75-86, (2006).

3. E.Ott, C.Grebogi, J.A.Yorke, Phys Rev Lett, 64(11), 1196-1199, (1990).

4. L.M.Pecora , T.L.Carroll, Phys Rev Lett , 64(8), 821-824, (1990).

5. J.H.Park , O.M.Kwon, Chaos, Solitons \& Fractals, 23(2),495-501,( 2005).

6. F.Wang, C.Liu, Physics Letters A , 360(2), 274-278, (2006).

7. A.E.Matouk, Communications in Nonlinear Science and Numerical Simulation, 16(2), 975-986, (2011).

8. X.Q.Wu, J.A.Lu , Chaos, Solitons \& Fractals, 18(4),721-729,(2003).

9. A.Khadra, X.Liu, X.Shen, IEEE Trans. On Circuits and Systems, 50(3),341-350, (2003).

10. H.T.Yau. Mechanical Systems and Signal Processing, 22(2), 408-418, (2008).

11. J.Lu , X.Wu , X,Han, J.Lú, Phys Lett A , 329,327333, (2004).

12. J.H.Park. Int J Nonlin Sci Numer Simulat , 6,201206, (2005).

13. J.Meng ,X.Y.Wang, Phys. Lett. A, 369(4): 294298,2007.

14. C.Li, X.F.Liao,W. Kwokwo , Physica D,194(3),187-202,(2004).

15. C.Masoller, H.Zanette, Physica A, 300,359366,(2001).

16. Y.W.Wang, Z.H.Guan, Chaos, Solitons \& Fractals,27,97-101,(2006).

17. N.F.Rulkov, M.M.Sushchik, L.S.Tsimring, H.D.I. Abarbanel, Phys Rev E,51,980 - 94, (1995).

18. M. Hu, Z.Xu, Nonlinear Analysis: Real World Applications, 9(3):1253-1260, (2008).

19. D.Ghosh, S. Banerjee, A. R. Chowdhury, Physics Letters A, 374(21),2143-2149, (2010). 